\title{
The use of exercise in mental health care
}

\author{
Ziogou Theologia $^{1}$, Fradelos Evangelos ${ }^{2}$, Kourkouta Lambrini ${ }^{1}$ \\ ${ }^{1}$ Department of Nursing, Alexander Technological Educational Institute of Thessaloniki, Thessaloniki, Greece \\ ${ }^{2} 3^{\text {rd }}$ Psychiatric Department, State Mental Hospital of Attica "Daphne", Athens, Greece
}

\section{Email address:}

thziogou@nurse.teithe.gr (T. Ziogou), evagelosfradelos@hotmail.com (E. Fradelos), laku1964@yahoo.gr (L. Kourkouta)

\section{To cite this article:}

Ziogou Theologia, Fradelos Evangelos, Kourkouta Lambrini. The Use of Exercise in Mental Health Care. American Journal of Nursing Science. Special Issue: Mental Health Care: Aspects, Challenges and Perspectives. Vol. 4, No. 2-1, 2015, pp. 16-21.

doi: 10.11648/j.ajns.s.2015040201.14

\begin{abstract}
Aim of the present research was planned to examine: a) the perceptions of professionals of mental health with regard to the use of physical activity when working with patients with psychiatric disorders and b) to study professionals' decisive factors for the promotion of physical activity in the working context. Material and Method: The sample of the research constituted of 173 professionals of mental health (men $\mathrm{N}=52$, women $\mathrm{N}=121$ ) from the state Psychiatric Hospital and other Mental health services in the area of The ssaloniki. Results: The Correlation Analysis showed that the intention is connected positively with the positive effect of physical activity $(r=.49)$, the role of personal physical exercise $(r=.46)$, the relation between physical and mental health $(\mathrm{r}=.33)$, weaker correlations were found with the negative effect of physical exercise $(r=.28)$ and with the convictions of control $(r=-.26)$.Regression Analysis showed that the professionals attitudes towards physical activity, as well as the role of personal physical exercise, constitute important predicting factors for their intention the promotion of physical activity (34\%).On the other hand, the promotion in their working place, was very small, since $76 \%$ of them could not promote any physical activity at all. Conclusions: The promotion of physical activity from the mental health professionals depends so much on their personal positive opinions for the physical activity, but also from their personal evaluation of behavior and the availability of resources.
\end{abstract}

Keywords: Physical Activity, Psychiatric Disorder, Planned Behavior, Mental Health Professionals, Convictions of Control

\section{Introduction}

Physical activity and exercise in general is of one of the main indicators for prevention purposes in both mental and physical health[1]. In ancient Greece, exercise played an important role in contemporary day to day life and constituted part of formal education. The ideal citizen would be fit in body and mature in both mind and soul [2]. The

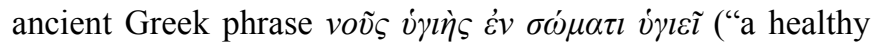
mind in a healthy body") used in antiquity means that a person's the mental state is closely related with his physical state [3]. Today scientific evidence supports that exercise is an important factor that contributes to mental balance and that mental health is closely related to physical exercise, the positive effects of which are being compared to those of psychotherapy. The above is supported by scientific research through which, an absolute correlation of a healthy mind and a healthy body is being shown [4]. Thus, exercise can function as a cure for the souls of millions of people in the world that face mental issues, like depression, contributing significantly to the resolution of said issues[5][6].

Exercise and physical activity have had a long standing relation with institutionalized psychiatric care and have played a great part in the cure of patients in the big mental hospitals built by the end of the 19th century [7]. Even though by now the majority of the big institutions that traditionally enclosed large areas for the recreation and occupation have been shut down and mental care is provided by smaller units within the community, physical activity often in the form of sports or exercise continues often to be included in the treatment programs being offered[8]. With regards to mental health professionals, they can be key players and participants in the healing process and the development of treatment programs. Thus physical exercise can be regarded as a valid and reliable addition to any therapeutic plan as also can the support of the individual's access in physical exercise regardless of whatever obstacles they may find its implementation [7][9]. 


\section{Aim}

The aim of this research has been to investigate the opinions of mental health professionals on the issue of physical activity and the main contributing factors in its promotion, or not, in treatment facilities.

\section{Material and Methods}

This research is based on the theory of planned behavior and examines the following variables: opinions on the relation between physical activity and mental health, attitude towards physical activity and exercise, opinions on the promotion of physical activity in the workplace, intentions for promotion on the workplace, beliefs on the evaluation of personal physical activity and obstacles for the promotion of physical activity[10][11].The participants of this study are mental health professionals working for the psychiatric hospital of Thessaloniki, facilities of mental health as well as psychiatric wards of the general hospital of Thessaloniki.

\subsection{Statistical Analysis}

Descriptive statistical analysis of the variables was conducted. The means, standard deviations and frequencies were obtained. Factor analysis (Varimax Rotation) was carried out on the two questionnaires that concerned (1) opinions on the relation between physical exercise and mental health and (2) the attitudes towards physical activity and exercise. Independent group t-tests where used for the investigation of differences in variables in this study as for the variables of sex and profession. Furthermore in order to test the study's hypotheses; correlation (Pearson's) and regression analyses, as well as analyses of variance were carried out. Statistical significance was set at $\mathrm{p}<.05$.

\subsection{Questionnaire}

Data was collected through questionnaires containing a total of 85 questions both open-ended and closed-ended. Questions were based theoretically on the model of planned behavior as well as the recommendations contained therein. All statements were graded using 5-point and 7-point Likert scales.

More specifically the structure of the questionnaires contained:

1 Demographics of the participants, such as sex, age, educational level, profession, experience and type of workplace.

2 Opinions on the relation between physical exercise and mental health (referring to the factors of attitude potential) [3]. These were evaluated through answers in a series of 20 questions. An example of a question would be "Physical activity and exercise take up an important part of my day to day life". Answers were given in a 7-point graded scale, ranging from 1: "I strongly agree ", to 7: "I strongly disagree". Internal consistency index Cronbach's Alpha for this scale was found to be .91 .
3 Attitude towards physical activity and exercise were evaluated through a series of 18 questions in a similar Likert scale of seven points spanning from 1:"I strongly agree" to 7:"I strongly disagree". Internal consistency index Cronbach's Alpha for this scale was found to be .89 .

4 Opinions on the promotion of exercise in the workplace were evaluated through a series of 9 questions in a similar Likert scale of seven points spanning from 1:"I strongly disagree" to 5:"I strongly agree".

5 Intensions of promoting physical activity in the workplace were evaluated using five statements graded on a 5-point Likert scale. An example would be "I intend to promote physical activity systematically"

6 Control Beliefs (conceived behavior control). Questions from this group totaled to a number of 13 and investigated the conceived control of the participants' behavior. They were worded in the following the form of: "How often do family commitments influence negatively your daily routine at work" and graded in a 5-point Likert scale. Internal consistency index Cronbach's Alpha for this scale was found to be 41 .

7 Evaluation of personal physical activity. This scale was measured based on the Godin "Leisure-Time Exercise Questionnaire" (Godin \& Shephard, 1986). This instrument contains 6 questions covering the type, frequency and duration of physical activities. For example, participants were asked "Do you engage in any form of moderate physical activity (like fast walking, running, or cycling) for a minimum of 20 minutes at least once a week?" Participants that answered "Yes", were subsequently asked for the specific weekly frequency and duration of their exercise. Answers were graded on a 6-point scale that ranged from 1 to 6 or more times a week. They mean duration was evaluated with a question between 20 minutes to 60 minutes or more.

8 Obstacles for the promotion of physical exercise. For the evaluation of the scale 8 questions were used of the following form "Which do you consider to be important obstacles for the promotion of physical exercise in your workplace: Spending on equipment/ Lack of trained staff. Answers when given in a 3-point scale ranging from 1: a significant obstacle, 2: not a valid obstacle and 3: not an obstacle at all.

\section{Results}

Questionnaires were filled out by 173 participants. The specimen was made up of 52 men (30.1\%) and 121 women (69.9\%) (Figure 1). The average age of the participants was 38.5 years (SD: 8.61 ). Ages 36 to 45 years represented $47 \%$ of the specimen, while $33.6 \%$ was younger than 35 years of age and $16.4 \%$ belonged to the $46-55$ age group. Among the participants, 114 were nurses, 29 were psychiatrists, 13 were clinical psychologists, 7 were social workers, 5 were occupational therapists, and 5 were trainers. A total of thirty 
participants did not record any profession information (Figure 2).

Participants were distributed between their workplace type: psychiatric hospitals $(n=117)$ units of rehabilitation $(n=14)$ mental health centers $(n=13)$ and psychiatric wards of general hospitals $(n=26)$. The educational levels of the participants were "Technical education" ( $\mathrm{n}=107)$, "Higher Education" (n $=52)$ and "Graduate" $(\mathrm{n}=11)$ and the average number of service time was 13.6 years $(\mathrm{SD}=7.7)$. . The $43.6 \%$ of the sample had work experience 11-20 years, $20.6 \% 5-10,18.8 \%$ had $<5$ years and $17 \%$ of $21-33$ years. (Average $=13.86$. SD $=7.7$ and a median of 15 . With regard to assessing the individual's physical activity results of the descriptive statistical analysis showed that $43.90 \%$ shows great interest in physical activity, 29\% little, 19,60\% significant and $7.50 \%$ none at all . Additionally $71 \%$ prefer brisk walking, cycling $13 \%, 8.4 \%$ running and $7.6 \%$ other forms of exercise Also, $50 \%$ exercises 2-3 times / wk., 16.20\% 4 times, $20.60 \%>4$ times and $13.20 \% 1$ once per week . $30.10 \%$ engages in exercise for $20 \mathrm{~min}, 29.40 \%$ for 30 , $28.7 \%>40$ minutes and $11.8 \%$ for 40 minutes at a time .

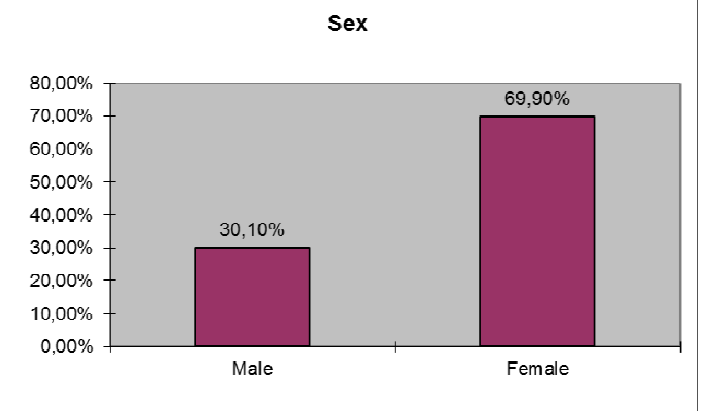

Figure 1. Sex distribution.

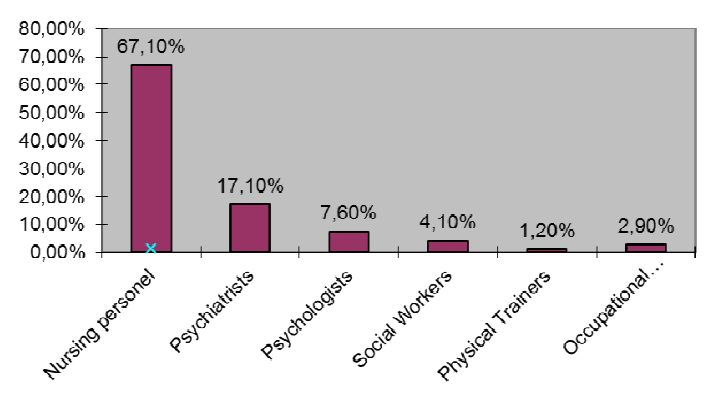

Figure 2. Profession
The type of physical exercise preferred is 60\% group activities and $40 \%$ individually .

All participants considered physical activity to be necessary in the treatment of persons with psychiatric disorders.More specifically, 98\% felt that their physical and mental health interact; $97 \%$ and $96 \%$ respectively considers exercise to be conducive to the mobilization, socialization and organization of time of persons with a psychiatric disorder; $94 \%$ believe that physical activity helps in selfcontrol and self-discipline and $93 \%$ that it helps wellbeing and self-esteem; $74 \%$ that it improves the quality of life of persons with psychiatric disorders .

Additionally regarding opinions for the promotion of exercise in the workplace, results showed that $75.70 \%$ did not sponsor any physical activity during the previous semester; $60.20 \%$ considers it very difficult to participate in any promotion; $61.30 \%$ said that there are similar initiatives on the premises; $57.90 \%$ stated that their colleagues do not participate in physical activity; $62 \%$ said that such an initiative is extremely valuable to them and $84.6 \%$ said they needed more information about the relation between physical activity and mental health. As many as $70.9 \%$ find their participation in physical activity useful, $62.6 \%$ beneficial, $48.9 \%$ feasible, $74.1 \%$ pleasant, relaxing $68.8 \%$ and $68.1 \%$ fun. Regarding their views on the main obstacles to the systematic application of exercise programs for people with mental disorders in open-ended questions variations and concerns about the mental state seem to arise . Regarding obstacles in promoting physical activity in work contexts results showed that $81 \%$ considered the lack of qualified personnel able to implement such programs, $68.2 \%$ the lack of knowledge relating to the planning, $64.7 \%$ equipment costs and $63.8 \%$ the lack of mobilization (motivation) of staff and patients. The absence of personal interest accounts for $47.4 \%$, the lack of time for $39.9 \%$ and poor personal fitness for $29.4 \%$.

\subsection{Descriptives}

The averages and standard deviations of the variables are presented in Table 1. The levels of internal consistency of the scales were high: Intent subscale $a=.78$, for the subscale "Relation between physical and mental health" $a=.94$, for the subscale "Role of exercise for each individual" $a=.71$, for the subscale "Positive effect of exercise" $a=.93$, for the subscale "negative effect of exercise" $\mathrm{a}=.87$, and the "control beliefs" subscale $a=.41$. (Table 1$)$. The range of all variables' values is from 1-5.

Table 1. Means, SD, Cron.a and correlations between variables of the theory of planned behavior:

\begin{tabular}{|c|c|c|c|c|c|c|c|c|c|}
\hline Variables & Mean & S.D & Cron. $\alpha$ & 1 & 2 & 3 & 4 & 5 & 6 \\
\hline 1. Intent & 2,45 & 0,68 & .78 & & & & & & \\
\hline 2. Relation between physical and Mental Health. & 1,89 & 0,87 & .94 & $.33 * *$ & & & & & \\
\hline 3. Role of exercise for each individual & 2,6 & 0,93 & .71 & $.46^{* *}$ & $0,54 * *$ & & & & \\
\hline 4.Positive effect of exercise & 2,27 & 1,21 & .93 & $.49 * *$ & $0,72 * *$ & $0,50 * *$ & & & \\
\hline 5.negative effect of exercise & 3,32 & 1,41 & .87 & $.28^{* *}$ & 0,07 & $-0,006$ & $0,20^{* *}$ & & \\
\hline 6.control beliefs & 2,88 & 0,44 & .41 & $-0,26$ & $-0,097$ & $-0,043$ & $-0,11$ & 0,03 & \\
\hline
\end{tabular}

$* \mathrm{P}<.05$

$* * \mathrm{p}<.001$ 


\section{Discussion}

The primary purpose of this study was to examine the beliefs on the use of physical activity on the mentally ill and the potentially determinant factors of promoting or not physical activity, based on the theory of planned behavior, among a sample of mental health professionals.

The results concerning the characteristics of mental health professionals showed that about half of the sample belongs to professionally active age groups and that the highest proportion were nurses. This is justified by the fact that we addressed hospitals. Moreover, the professionals surveyed represented proportionally their occupation and employment context. Although the selection of the sample was not based on strict sampling criteria, we believe the sample is largely representative and, thus, we can draw useful conclusions on the issue of the opinions of mental health professionals on promoting physical activities [4].

The frequency of and interest in personal physical activity was high. Half of our sample reported they exercised 2-3 times a week and $30 \%$ for at least 30 minutes. Our results agree with those of, but is higher than other studies that have examined the practices of physical training of health professionals and that of the general US population, and this is probably due to the fact that our sample was interested in exercise [9][13].

Above all, our results confirmed our first research hypothesis concerning the contribution of physical activity to better treatment of people with psychiatric disorders. More specifically, everyone believes that it is necessary for the therapeutic approach of these people. Specifically, as to the beneficial results, about $95 \%$ believe that it increases selfesteem, enhances social relationships and generally promotes physical and mental health. Our results agree with those of Burks \& Keeley (1989), where although professionals have a favorable attitude toward exercise for people with mental disorders and they engaged in physical activities themselves, few recommend exercise to their clients. From the results of our study, $75 \%$ did not sponsor any physical activity in the context of employment during the previous six months, despite the positive opinions of the majority of the role of exercise [14].

$84.6 \%$ said they needed more information about the relationship between physical activity and mental health. Our results agree with the existing literature reports showing the gap of knowledge held by health professionals despite a growing evidence base on the benefits of physical activity on physical and mental health [15]. Unfortunately, there is little evidence of research on physical activity in the field of mental health. We believe that a systematic dialogue with mental health professionals to assess the complex interactions of physical and mental part has never been initiated. Gauvin and Spence (1995) for example, note that in this region, "the infrastructure necessary for the translation of research to practice does not exist" [16].

In our study, $61.30 \%$ said that there are no similar initiatives in their respective workplace. For any promotion of physical activity to be successful, it is necessary to institutionalize a broad exercise routine. It is certain that a level of institutional support is necessary to legitimize the promotion of physical activity and strengthen the promotional behavior. Without this support, any intentions for the promotion and implementation can be neither realistic nor practical [17]. Promoting physical activity is a function of the rules of personal physical activity of the participants, as revealed by the results of our research which are consistent with those of McKenna et al. (1998) [18].

Participants identified several barriers to promoting physical activity in the workplace. The lack of qualified personnel and expertise, the equipment costs and the lack of mobilization (motivation) were among the most common perceived barriers. The lack of personal interest, poor personal fitness and lack of time, was one of the least mentioned obstacles. Mostly, the obstacles identified by professionals can be successfully addressed. For example, regarding the low motivation relevant literature suggests that interventions that focus on the mobilization of participants, can be useful [19][20].

Additionally, time management skills and concise training on less expensive types of physical activity (e.g., jogging, walking, cycling, video classes) are likely to increase the interest and willingness to participate in the promotion of physical activity. Moreover, the knowledge of those obstacles based in the opinion of the participants can attribute to the development of physical exercise interventions that are aware of the obstacles and can promote the highly participation of the professionals. [21].As to the second hypothesis, the intention to promote physical activity was predicted by attitudes toward the behavior (positive or negative), and the role of physical activity for each individual. A number of studies have shown that attitude towards behavior predicts intention more than the subjective norm does and that there is a relationship between positive attitudes and engaging in behavior[22].The mental health professionals who participated needed to have a more positive view of the impact of physical activity and engage in regular exercise themselves in order to promote physical activity. This agrees with the results of McKenna et al. (1998) and Mc Entee et al. (1996), where promotion of physical activity is associated with personal rules of physical activity of participants [18][23].

Also, the intention was significantly correlated with attitudes toward the behavior and general views about the role of exercise in the same person, and a negative correlation with the control beliefs. This could clearly relate to the type of behavior being evaluated, since when considering general attitudes concerning work, the perceived usefulness of behavior can have a greater impact on beliefs control behavior. Our results do not agree with those of Bunce et al., (1998) argue that the primacy of belief monitoring in predicting the intention of health professionals[24].

Although control beliefs are discussed as important for the prediction of intention, in our study another number of 
factors appears to affect intent (such as lack of knowledge, expertise, lack of structures, professional commitments, etc.). According to the theory of planned behavior when the actual behavioral control is high, control beliefs have only an indirect effect [4]. This could clearly concern the type of behavior being evaluated. In consideration therefore of workrelated behaviors, the perceived usefulness of behavior can have a greater impact on perceived performance review of the conduct and the intention is sufficient to predict participation in the promotion of physical activity [15]. In the development of intentions, interventions should facilitate perceptions of professionals to control the behavior as it affects the formation of promotional intent. This would involve the disclosure and examination of existing protocols for promoting physical activity (e.g., PACE program and PAL program) and supply professionals with the skills to promote physical activity in a way that can be integrated to their existing roles. While positive views towards the promotion may be a condition, it is possible that beliefs control is essential for the translation of positive intentions into action. Consequently, it is likely that increased intentions to promote physical activity through a series of educational seminars designed especially in order to change their views and strengthen their beliefs control [4].

The terms of interventions, such as the development of training programs at undergraduate and postgraduate level could actually lead to convergence between physical and mental factors for a holistic model of health. Practically, surveys suggest that professionals need help to develop plans to promote physical activity. A specific protocol that specifies what should be promoted and by whom [25]. Experienced researchers believe that there are "signs" that health professionals can contribute to upgrading promotion [26]. The third research hypothesis concerned the differences between the sexes in the research variables. The results showed that men had more positive attitudes to the role of physical activity for themselves. Here, there were no differences in the variables of intention, attitudes towards the behavior of the potential of attitudes and beliefs control. And as to the differences between the occupations observed that OT as well as gymnasts promote physical activity more than other professionals, and this is probably related to the nature of the profession [4].

\section{Conclusions}

Promotion of physical activity by mental health professionals appears to be a function of stronger intentions, more positive views of the role of exercise on the person and prerequisites. Understanding the factors that contribute to the promotion of physical activity, the theory of planned behavior may be a useful tool for promoting physical activity within departments that support health care and theoretically support interventions to increase the participation of professionals in such behaviors.

Considering also that there are still numerous research paths that could shed light on the subject of this study further, we it would useful to examine in greater detail the beliefs and views of the therapeutic role of exercise and how exercise issues could be integrated into education programs generally.

\section{References}

[1] Kafkia T, Zinelis D, KourakosM.Exercise Protocols in Patients with Chronic Kidney Disease (CKD) andTherapy:A Literature Review on Renal Replacement .Hellenic Journal of Nursing Science 2008; 1 (2): 56-59

[2] Kourkouta L., Elafri M., Tsiotsiou V., Strogkylou V. Exercise and Mental Health in Ancient Greece. 40 Psychiatric Conference on Primary Health Care. Kyllini Ilia 1- 04.06.2012.

[3] Kourkouta L., Plati P., Procopiou E.: A healthy mind in a healthy body. 390 Panhellenic Nursing Conference. Volos, 1518 May in 2012.

[4] Ziogou Th. The views of mental health professionals on the effects of physical activity in people with mental disorders and their participation or in the non-promotion of the care centers. Thesis. Department of Physical Education and Sport Science, Aristotle University of Thessaloniki.Thessaloniki, 2006.

[5] Tsaousoglou A KoukourikosK. Quality and healthservices.Stigma, 2007; 15 (1): 18 -24.

[6] Ziogou Th, Kourkouta L, Diktapanidou S, Fradelos E, Ouzounakis P. Exercise care of mentally asthenon.3o Congress Biopsychosocial Approach to Health Care. With International Participation.Thessaloniki, 28-30 March2013.

[7] Fox K R. The influence of physical activity on mental wellbeing. Public Health and Nutrition, 1999; 2: 411- 418.

[8] Hays K.F. (1999) Working It Out: Using Exercise in Psychotherapy. American Psychological Association, Washington DC.

[9] Faulkner G. \& Biddle S. (2001a) Exercise as therapy: it's just not psychology! Journal of Sports Sciences 19, 433-444

[10] Ajzen, I. (1988). Attitudes, personality and behavior. Chicago: Dorsey Press.

[11] Ajzen, I.(2002) Constructing a TpB questionnaire: conceptual and methodological considerations.[ On Line] Available : http://www-nix.oit.umass.edu/ aizen/

[12] Godin, G., \& Shephard, R.(1986a). Importance of type of attitude to the study of exercise-behaviour.Psychological Reports, 58, 991-1000.

[13] Stephens, T.(1988) Physical activity and mental health in the United States and Canada: Evidence from four population surveys. Preventive Med,17:35-47

[14] Burks, R., Keeley, S. (1989). Exercise and diet therapy: Psychotherapists' beliefs and practices. Professional Psychology: Research and Practice, 20, 62-64.

[15] Gould MM, Thorogood M, Illifle S, Morris JN. (1995). Promoting physical activity in primary care: measuring the knowledge gap. Health Educ J., 54, 304-311.

[16] Gauvin L, Spence JC. (1995). Psychological research on exercise and fitness: current research trends and future challenges. Sport Psychol., 9, 434-448. 
[17] Kreuter M., Scharff D., Brennan L., Lukwago S.(1997). Physisian recommendations for diet and physical activity: which patients get advised to change? Prev. Med., 26, 825-833.

[18] McKenna, J., Naylor, P.\& McDowell, N. (1998). Barriers to physical activity promotion by GPs and practice nurses. British Journal of Sports Medicine, 32, 242-247.

[19] Yahne, C.E. and Miller (1999). Enhancing motivation for treatment and change. In: Mc Crady, B.S. and Epstein, E., Editors, 1999. Addictions: a comprehensive guidebook, Oxford University Press, New York, 235-249.

[20] Walitzer K.S., Derman K.H. and Connors G.L. (1999). Strategies for preparing clients for treatment: a review. Behavior Modification., 23, 129-151.

[21] Lynch, D., Repka, F., Nagel, R., Birk, T., Gohara, A., Leighton, R., Walsh, M., \& Weaver, M.,(2000). Prediction of dietary adherence in cholesterol reduction: relative contribution of personality variables and health attitudes. Psychology and Health 15, 821-828.
[22] Theodorakis, Y., Natsis, P., Papaioannou, A., \& Goudas, M.(2003). Greek students' attitudes toward physical activity and health- related behaviour. Psychological Reports, 92, 275283.

[23] Mc Entee, D., \& Halgin, R. (1996).Therapists' attitudes about addressing the role of exercise in psychotherapy.Journal of Clinical Psychology, 52, 48- 60.

[24] Bunce D, Birdi K. (1998). The theory of reasoned action and theory of planned behaviour as a function of job control. Br J Health Psychol., 3, 265-275.

[25] Stokols D. (1992). Establishing and maintaining health environments: toward a social ecology of health promotion. Am Psychol., 47, 6-22.

[26] Simons-Morton DG, Calfas KJ, Oldenburg B, Button N. (1998). Effects of interventions in health care settings on physical activity or cardio respiratory fitness.Am J Prev Med., $15,413-430$. 